\title{
Preliminary experience of fractionated stereotactic radiosurgery with extend system of Gamma Knife
}

\author{
Raj Kishor Bisht' ${ }^{1}$, Shashank Sharad Kale ${ }^{1}$, Gopishankar Nathanasabapathi' ${ }^{1}$ Pratik Kumar', \\ Manmohan Jit Singh ${ }^{1}$, Deepak Agarwal', Sanjay Thulkar'3, Ajay Garg', Pramod Kumar Julka ${ }^{5}$, \\ Gaura Kishor Rath ${ }^{5}$, Bhawani Shankar Sharma ${ }^{1}$
}

\author{
${ }^{1}$ Department of Neurosurgery and Gamma Knife, All India Institute of Medical Sciences, New Delhi, India \\ ${ }^{2}$ Department of Medical Physics (BRAIRCH), All India Institute of Medical Sciences, New Delhi, India \\ ${ }^{3}$ Department of Radiodiagnosis (BRAIRCH), All India Institute of Medical Sciences, New Delhi, India \\ ${ }^{4}$ Department of Neuroradiology, All India Institute of Medical Sciences, New Delhi, India \\ ${ }^{5}$ Department of Radiotherapy, All India Institute of Medical Sciences, New Delhi, India
}

Received November 09, 2015; Revised February 11, 2016; Accepted February 12, 2016; Published Online February 16, 2016

\section{Original Article}

\begin{abstract}
Purpose: The purpose of this study is to present multisession stereotactic radiosurgery with initial experience using custom made extend system (ES) of Gamma Knife. Methods: The ES is comprised of a carbon fiber frame also called extend frame, vacuum head rest cushion, patient surveillance unit and a configurable front piece with dental impression tray. The extend frame is a rigid connection between patient's head and patient positioning system (PPS) of Gamma Knife. A dental impression of patient was created and attached to the frontal piece of extend system. The treatment setup involves positioning the patient within the extend frame using patient specific headrest cushion and front piece. The reference patient's head position was recorded through measurements of repositioning check tool (RCT) apertures using a high precision digital probe before computed tomography (CT) scan. The RCT measurements taken before treatment were compared with recorded reference position to ensure appropriate patient treatment position. Volumetric magnetic resonance (MR) scan was co-registered with stereotactic CT scan on Leksell Gamma plan. Fused MR to CT images on Gamma Plan was utilized to delineate regions of interest and prepare a precise treatment plan. The presented study includes positional reproducibility check and dosimetric evaluation of ten patients treated with ES. Results: Forty-three fractions on ten patients with prescribed treatment format were delivered successfully. An average tumor volume of $11.26 \mathrm{~cm}^{3}$ (range, $340 \mathrm{~mm}^{3}$ to $59.12 \mathrm{~cm}^{3}$ ) was treated with ES. The mean tumor coverage of $91.91 \%$ (range, 90\% to 95\%) was able to achieve at $50 \%$ prescription isodose without compromising adjacent normal structure radiation dose tolerances. The mean inter-fraction positional variation of $0.69 \mathrm{~mm}$ influences an inherent strength of immobilization technique. Follow-up of seven patients at a median interval of 16 months (range, 9 months to 26 months) showed evidence of $100 \%$ radiographic control with improved clinical results. Conclusion: Conjugative clinical outcome shows the efficacy of fractionation in various clinical indications.
\end{abstract}

Keywords: Gamma Knife; Stereotactic Radiosurgery; Positional Accuracy

\section{Introduction}

Gamma Knife stereotactic radiosurgery with single high radiation dose fraction offers an efficient treatment to a number of clinical indications, such as arteriovenous

malformation, brain tumor, trigeminal neuralgia, epilepsy, Parkinson's disease and intractable pain. ${ }^{1-5}$ Treating target volume with relatively lower radiation 
dose fractions have radiobiological advantage in increasing radiobiological effect on tumor and sparing adjacent normal tissue..$^{5-9}$ Previous studies of multisession stereotactic radiosurgery with Gamma Knife machine required repeated application of Leksell coordinate base frame or prolonged period of frame application. ${ }^{10}$ These techniques ensure treatment ethics, however, the quality and acceptance of such treatments is questionable. In addition, the clinical complication rate belies the stereotactic treatment aim.

The extend system based fractionated treatment delivers a radiation dose in multiple fractions. ${ }^{11}$ Enhanced Gamma plan software is being used to prepare a conformal treatment plan and compare radiological treatment outcomes. Physical parameters and quality indices of a precise treatment plan were evaluated with clinical outcomes of ten patients treated for various clinical indications. The presented study is preliminary experiences of multi-session stereotactic radiosurgery with extend system on Gamma Knife Perfexion. In this study, we investigated the potential of custom made extend system with improvised positional shift calculations.

\section{Methods and Materials}

The extend System (ES) enables fractionated stereotactic radiosurgery with Leksell Gamma Knife Perfexion (ELEKTA, AB Stockholm, Sweden). A reproducible patient treatment setup is core component of multi-session stereotactic radiosurgery. The patient docking device used for single session stereotactic radiosurgery (SRS) with Gamma Knife is replaced with extend frame in fractionated treatments (Figure 1a). ${ }^{\mathbf{1 0 - 1 1}}$ The extend frame is a rigid connection between patient's head and patient positioning system (PPS) of Gamma Knife Perfexion.

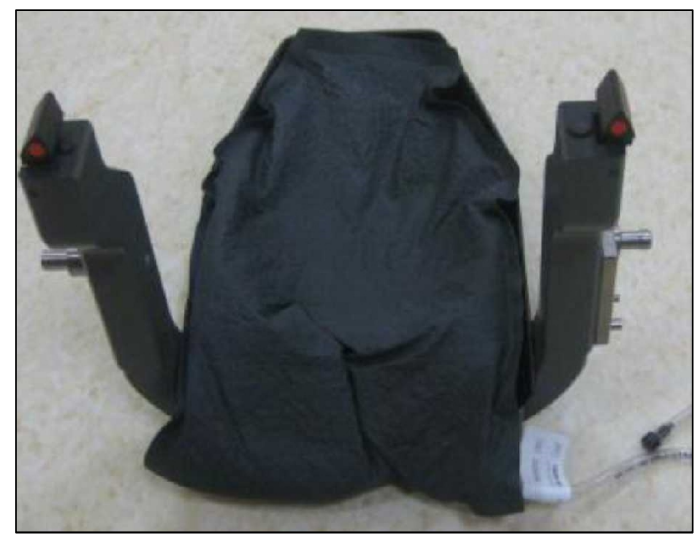

Figure 1 (a): Carbon- fiber extend frame and vacuum head rest cushion.

\subsection{System description}

The ES is comprised of a carbon fiber frame or extend frame, vacuum head rest cushion, patient surveillance unit (PSU) and a configurable front piece with patient specific mouth bite. The extend frame is docked to the PPS of Gamma Knife at a head angle of 90 degrees. The front piece of extend system includes $\mathrm{X}-\mathrm{Z}$ sliding plate, $\mathrm{a}$ fixed top plate, clamp and securing screws. The mouth bite with dental impression tray is attached to the clamp of front piece (Figure $1 \mathrm{~b}$ ). Preparation of patient specific mouth bite and vacuum assisted mouth piece fixation with frontal plate assembly is mentioned in Sayer et al. ${ }^{11} \mathrm{~A}$ vacuum cushion on extend frame holds the patient's head in position during imaging and treatment. A soft head cushion turns into rigid and head shaped on applying a suction of $66 \%$ to $70 \%$ on PSU. The patient surveillance unit consists of a vacuum pump, surveillance system, digital probe connections and a patient alert alarm (Figure $1 \mathrm{c}$ ). For the most optimal comfort and patient safety, a constant vacuum level between $40 \%$ and $45 \%$ on PSU, found appropriate to keep patient mouth bite intact during imaging and treatment (indicative suction/vacuum level values are related to ambient atmospheric pressure) (Table 1). The combination of patient specific vacuum head rest cushion and frontal plate assembly has to remain unchanged until completion of the treatment.

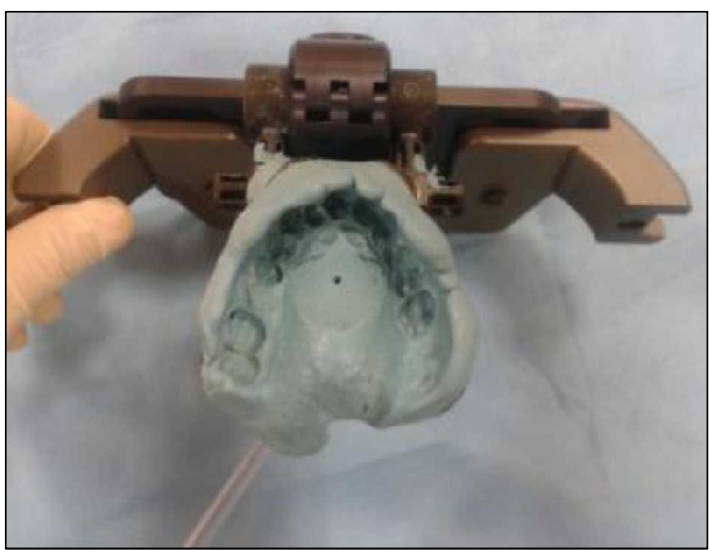

Figure 1(b): Front piece of extend system with patient dental impression and suction tube.

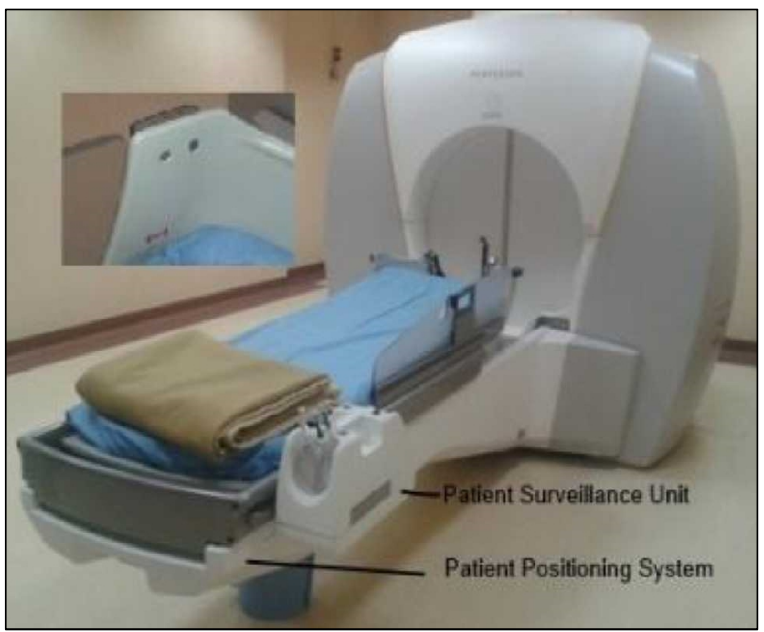

Figure 1(c): Patient positioning system and patient surveillance unit. 
Table 1: Suction level on PSU (a) head rest preparation; (b) during patient treatment.

\begin{tabular}{cc|ccc}
\hline \hline Cases & $\begin{array}{c}\text { Relative suction (\%) } \\
\text { Vacuum cushion } \\
\text { preparation (a) }\end{array}$ & \multicolumn{2}{c}{$\begin{array}{c}\text { Relative suction (\%) } \\
\text { During treatment (b) }\end{array}$} \\
\cline { 4 - 5 } & 68 & 41 & 40 & 41 \\
1 & 69 & 43 & 43 & 43 \\
2 & 70 & 40 & 40 & 40 \\
3 & 70 & 45 & 44 & 45 \\
4 & 66 & 44 & 45 & 45 \\
5 & 70 & 43 & 44 & 44 \\
6 & 68 & 42 & 41 & 43 \\
7 & 66 & 45 & 45 & 45 \\
8 & 70 &
\end{tabular}

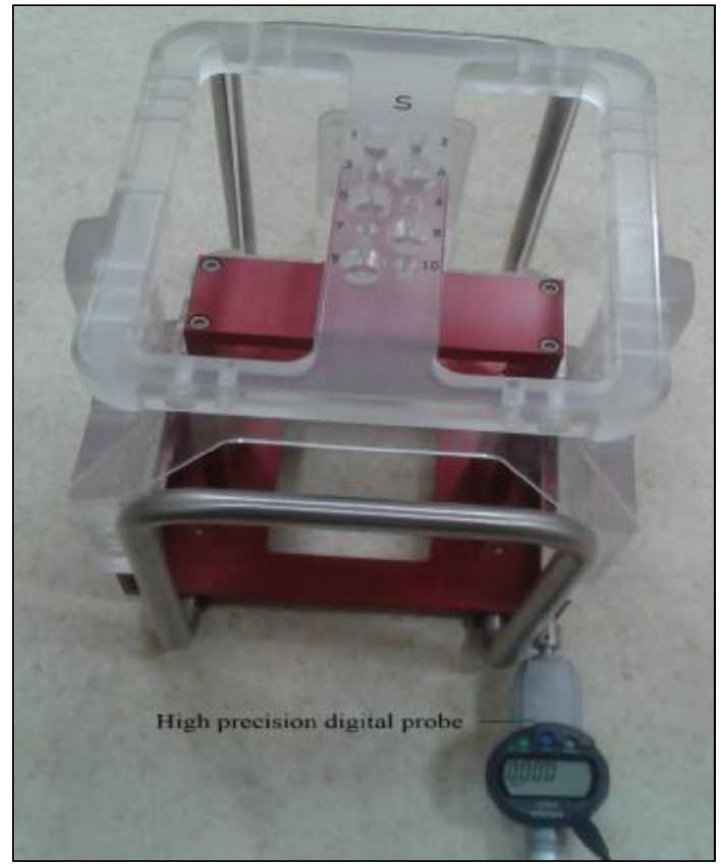

Figure 2: Repositioning check tool (RCT) and calibration of electronic digital probe.

The patient's head position within the extend frame is estimated using repositioning check tool (RCT) measurements (Figure 2). A calibrated spring tip digital measurement probe (Absolute Digimatic Model ID-C150XB, Mitutoyo Corp, Kawasaki, Japan) is used to measure encoded RCT apertures (Figure 2). Long probe was utilized throughout the presented study, which was calibrated using L0-L1-L0 protocol before treatment. The L0-L1-L0 protocol is "setting the correct zero" position of a spring tip digital probe. Repeated measurements of the L1 labelled hole provided at RCT quality assurance tool followed by "zero setting" at the L0 labelled hole were observed to check probe consistency.

\subsection{Determination of reference values}

The treatment position is conserved through recording of suitable RCT aperture depths. The RCT is able to mount over the patient's head on extend frame assembly. The RCT has 44 distinct encoded apertures arranged in four side panels. The apertures for which the digital probe tip falls perpendicular to the patient surface were chosen to prepare reference data sheet. Curved surface points or the aperture closed to head surface were ignored while selecting reference apertures on RCT. The reference data sheet corresponds to the patient's head position for consecutive fractions at PPS of Gamma Knife. The positional accuracy and the prominence of reproducibility during treatment were evaluated by comparing reference data sheet and the RCT measurement across fractions. The reference values are recorded before patient scan on the CT couch (Figure 3); whereas, an average of three level measurements i.e., (i) primarily on PPS of Gamma Knife (ii) before and (iii) after stereotactic CT image acquisition, was preferred to prepare reference data sheet in this study. Similarly, maximum possible apertures (Table 2) were recorded to evaluate patient positioning error or radial difference vector.

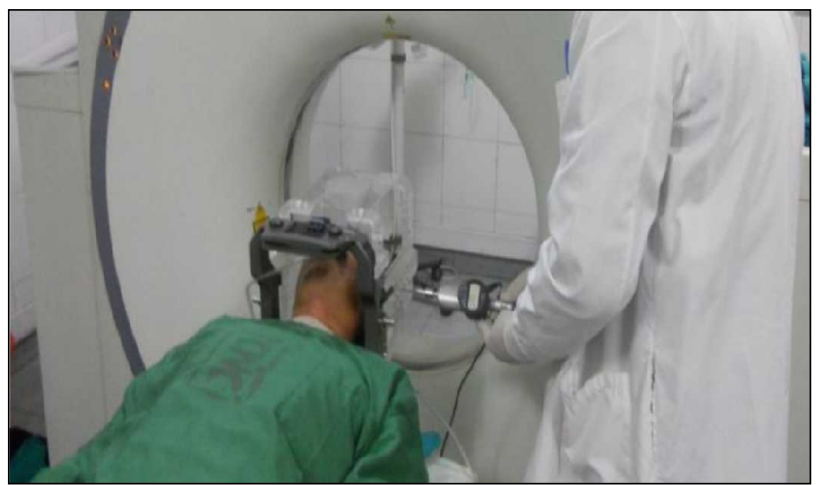

Figure 3: Recording of reference data sheet on CT couch.

Table 2: Selected RCT measurement points per panel for patient positioning.

\begin{tabular}{cccccc}
\hline \hline S. N & $\begin{array}{c}\text { No. of } \\
\text { measured } \\
\text { A }\end{array}$ & $\begin{array}{c}\text { No. of } \\
\text { measured } \\
\text { B }\end{array}$ & $\begin{array}{c}\text { No. of } \\
\text { measured } \\
\text { C }\end{array}$ & $\begin{array}{c}\text { No. of } \\
\text { measured } \\
\text { D }\end{array}$ & $\begin{array}{c}\text { No. of } \\
\text { measured } \\
\text { E }\end{array}$ \\
\hline 1 & 16 & 4 & 4 & 4 & 4 \\
2 & 12 & 3 & 3 & 3 & 3 \\
3 & 12 & 3 & 3 & 3 & 3 \\
4 & 15 & 4 & 4 & 4 & 3 \\
5 & 16 & 4 & 4 & 4 & 4 \\
6 & 18 & 7 & 4 & 3 & 4 \\
7 & 19 & 5 & 5 & 5 & 4 \\
8 & 19 & 5 & 5 & 5 & 4 \\
9 & 18 & 5 & 4 & 4 & 5 \\
10 & 14 & 3 & 4 & 4 & 3 \\
\hline
\end{tabular}

$\mathrm{A}=$ RCT apertures; $\mathrm{B}=$ Apertures on left panel of RCT; $\mathrm{C}=$ Apertures on right panel of RCT; D = Apertures on anterior panel of RCT; E = Apertures on superior panel of RCT.

\subsection{Radial difference vector (RDV)}

The reference values were entered into the treatment console before the first fraction of the multisession treatment and compare with measured values before consecutive fractions. The system calculates and 
displays the resultant radial difference vector (RDV) on treatment console when all apertures are measured (Table 3). Positional shift or radial difference vector $(\Delta \mathrm{d})$ is calculated using a simple statistical formula:

$$
\Delta \mathrm{d}=\sqrt{\left(\delta \mathrm{x}^{2}+\delta \mathrm{y}^{2}+\delta \mathrm{z}^{2}+\delta \mathrm{s}^{2}\right)}
$$

Where; $\delta \mathrm{x}, \delta \mathrm{y}$ and $\delta \mathrm{z}$ are mean positional shifts along the $\mathrm{x}, \mathrm{y}$ and $\mathrm{z}$-axes. We have assumed " $\delta \mathrm{s}$ " is representative of mouth bite shift shown in Figure 4 (a \& b).

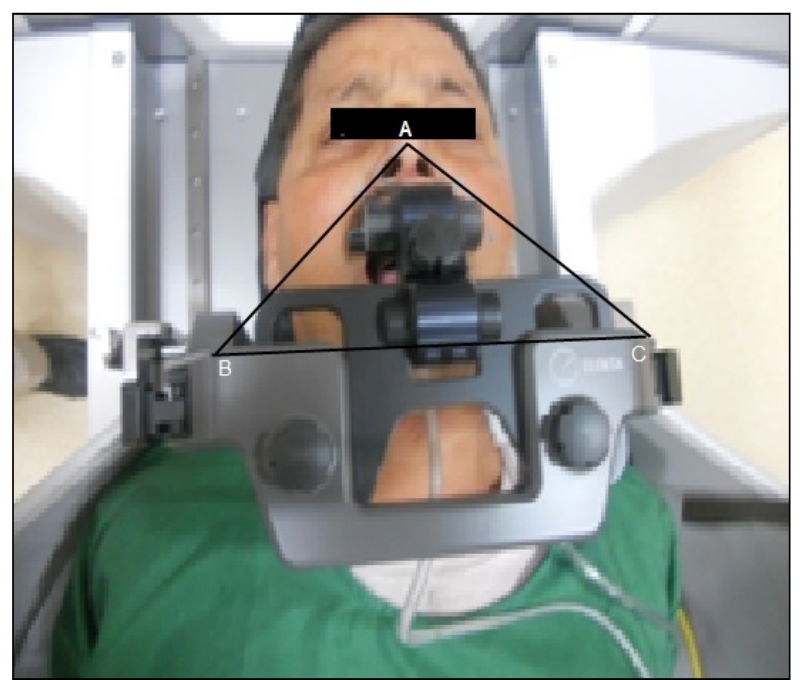

Figure 4 (a): Determination of mouth bite shift $(\delta s)$.

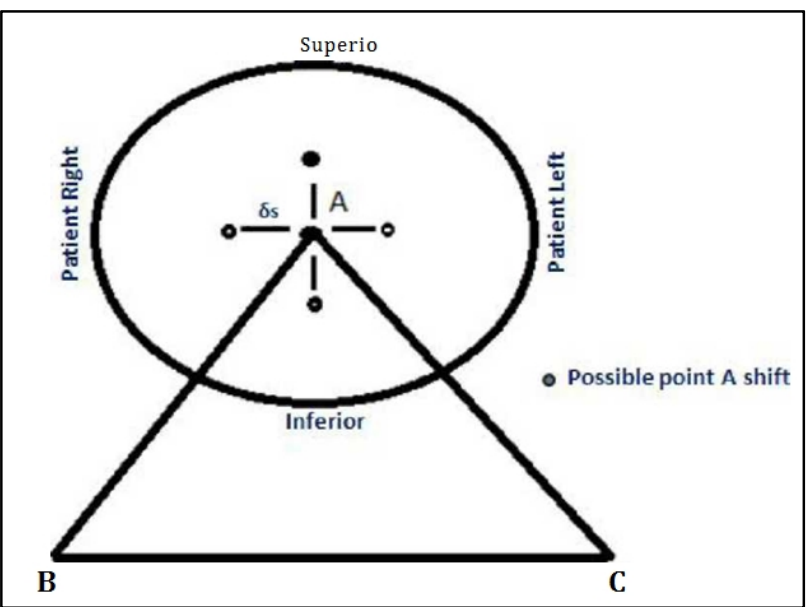

Figure 4 (b): Possible point "A" shift in vertical and horizontal planes.

\subsection{Patient imaging}

For fractionated treatment with ES; the regions of interest were localized using acquired stereotactic CT images, whereas non stereotactic volumetric MR images were fused using co-registration tool on treatment planning system. ${ }^{12}$ The non-contrast stereotactic CT images were obtained using parameters as follows: Tube Voltage - $120 \mathrm{kVp}$, Tube current - $100 \mathrm{mAs}$, slice thickness $-1 \mathrm{~mm}$ and FOV $-230 \mathrm{~mm} \times 230 \mathrm{~mm}$. The tumor and critical structures or organs at risk were delineated on fused or clinically relevant image sequences.

Table 3: RDV per fraction and comparison between system generated and manually calculated RDV.

\begin{tabular}{|c|c|c|c|c|c|}
\hline S. N & $\begin{array}{c}\text { No. of } \\
\text { Fractions }\end{array}$ & $\begin{array}{l}\text { Radial difference vector } \\
\text { (RDV in mm) }\end{array}$ & $\begin{array}{c}\text { Average RDV } \\
(\mathrm{mm})\end{array}$ & $\%$ Variation & $\begin{array}{l}\text { RDV with measurement of } \\
12 \text { RCT apertures (mm) }\end{array}$ \\
\hline \multirow[t]{2}{*}{1} & 5 & A: $1.00,0.90,0.70,0.40,0.70$ & 0.740 & & - \\
\hline & & M: $0.97,0.90,0.68,0.42,0.71$ & 0.736 & 0.54 & \\
\hline \multirow[t]{2}{*}{2} & 5 & A: $1.50,0.60,0.70,0.60,0.70$ & 0.820 & & - \\
\hline & & $\mathrm{M}: 1.45,0.58,0.68,0.62,0.72$ & 0.810 & 1.21 & \\
\hline \multirow[t]{2}{*}{3} & 5 & A: $0.80,0.50,0.60,0.60,0.50$ & 0.600 & & - \\
\hline & & M: $0.82,0.52,0.58,0.60,0.50$ & 0.604 & 0.66 & \\
\hline \multirow[t]{2}{*}{4} & 4 & A: $0.80,0.70,0.60,1.00$ & 0.780 & & - \\
\hline & & M: $0.81,0.73,0.62,0.96$ & 0.780 & 0.00 & \\
\hline \multirow[t]{2}{*}{5} & 4 & A: $0.30,0.90,0.90,0.50$ & 0.650 & & - \\
\hline & & M: $0.28,0.89,0.92,0.50$ & 0.648 & 0.31 & \\
\hline \multirow[t]{2}{*}{6} & 4 & A: $1.20,0.50,1.00,1.10$ & 0.950 & & \\
\hline & & $\mathrm{M}: 1.21,0.48,0.96,1.14$ & 0.948 & 0.21 & 0.988 \\
\hline \multirow[t]{2}{*}{7} & 4 & A: $0.50,0.80,0.80,0.80$ & 0.720 & & \\
\hline & & M: $0.52,0.80,0.81,0.77$ & 0.725 & 0.69 & 0.771 \\
\hline \multirow[t]{2}{*}{8} & 4 & A: $0.30,0.40,0.50,0.60$ & 0.450 & & \\
\hline & & M: $0.29,0.41,0.48,0.60$ & 0.445 & 1.11 & 0.521 \\
\hline \multirow[t]{2}{*}{9} & 4 & A: $0.50,0.50,0.70,0.80$ & 0.620 & & \\
\hline & & M: $0.53,0.51,0.68,0.78$ & 0.625 & 0.80 & 0.651 \\
\hline \multirow[t]{2}{*}{10} & 4 & A: $1.00,0.30,0.60,0.70$ & 0.650 & & \\
\hline & & M: $0.96,0.31,0.62,0.70$ & 0.647 & 0.46 & 0.760 \\
\hline
\end{tabular}

$\mathrm{A}=$ Auto generated RDV; $\mathrm{M}=$ Calculated RDV 


\subsection{Patient imaging}

For fractionated treatment with ES; the regions of interest were localized using acquired stereotactic CT images, whereas non stereotactic volumetric MR images were fused using co-registration tool on treatment planning system. ${ }^{12}$ The non-contrast stereotactic CT images were obtained using parameters as follows: Tube Voltage - $120 \mathrm{kVp}$, Tube current - $100 \mathrm{mAs}$, slice thickness $-1 \mathrm{~mm}$ and FOV $-230 \mathrm{~mm} \times 230 \mathrm{~mm}$. The tumor and critical structures or organs at risk were delineated on fused or clinically relevant image sequences.

\subsection{Treatment planning and quality indices}

Leksell Gamma Plan (LGP) version 10.1 hosted on a PC platform with Linux operating system is used to evaluate fractionated stereotactic radiosurgery treatments. A range of planning tools on LGP is being used to create a precise treatment plan on DICOM supported images obtained and transferred from CT, MR and angiography units. Patient skull contouring was done using stereotactic CT images on LGP. The algorithms embedded within the Leksell Gamma Plan and available planning tools provide the cumulative radiation dose distribution in the radiation field. The treatment regimen is developed and followed by various available data for single fraction stereotactic radiosurgery and

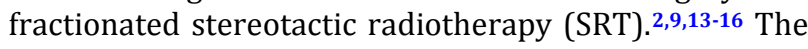
quality indices of treatment plan were calculated as tumor coverage (C), conformity index (CI), selectivity (S) and gradient index (GI) using equations $2 \mathrm{a}-\mathrm{d}$.

$$
\begin{aligned}
& \mathrm{C}=\{(\mathrm{PIVp} \cap \mathrm{TV}) /(\mathrm{TV})\} \\
& \mathrm{CI}=\{(\mathrm{PIVp}) /(\mathrm{TV})\} \\
& \mathrm{S}=\{(\mathrm{PIVp} \cap \mathrm{TV}) /(\mathrm{PIV})\} \\
& \mathrm{GI}=\{(\mathrm{PIVp}) /(\mathrm{PIVp} / 2)\}
\end{aligned}
$$

Where; PIVp $=$ prescription isodose volume, PIVp $/ 2=$ half of the prescription isodose volume

\section{Results}

Following fractionated stereotactic radiosurgery regimen, the patients were treated with 4 - 5 relatively lower radiation dose fractions (Table 4). All fractions with prescribed treatment format were delivered successfully.

\subsection{Positional accuracy check}

On application of L0-L1-L0 calibration protocol, the long digital probe was found suitable for repeated measurements with an accuracy of $0.0026 \mathrm{~mm}$. The maximum number of possible RCT apertures (average, 16 encoded points) has been measured to prepare reference position data sheet (Table 2). The radial difference vector, generated by the system shows an average patient shift of $0.69 \mathrm{~mm}$ (Standard deviation of $0.14 \mathrm{~mm}$ ) from the reference patient position across all patients and fractions. Post fraction RCT measurements shows the mean intra-fraction positional shift across all treatments was $0.33 \mathrm{~mm}$ (SD of $0.12 \mathrm{~mm}$ ).

\subsection{Volumetric evaluation}

The volumetric evaluation on resultant histogram is a measure of absolute/relative doses received into the delineated volumes. Forty-three fractions on eight male and two female patients of different clinical indications were delivered through fractionated radiosurgery regimen. An average tumor volume of $11.26 \mathrm{~cm}^{3}$ (range

\begin{tabular}{|c|c|c|c|c|c|c|c|c|}
\hline S.N & Age/Sex & Indication & $\mathrm{TV}(\mathrm{cc})$ & $\begin{array}{c}\text { Dose per fraction } \\
\times \text { Fractions } \\
\end{array}$ & $\mathrm{CI}$ & $\mathrm{S}$ & GI & $\mathrm{mTD} \%$ \\
\hline 1 & $50 / \mathrm{M}$ & Meningioma & 10.39 & $4 \mathrm{~Gy} \times 5$ & 1.21 & 0.82 & 2.67 & 19.25 \\
\hline 2 & $52 / \mathrm{M}$ & Pituitary Adenoma & 8.06 & $5 \mathrm{~Gy} \times 5$ & 1.46 & 0.84 & 3.12 & 21.40 \\
\hline 3 & $28 / \mathrm{M}$ & AVM & 0.340 & 5 Gy $\times 5$ & 2.40 & 0.76 & 2.98 & 44.40 \\
\hline 4 & $62 / M$ & Pituitary Adenoma & 16.04 & 3.5 Gy × 4 & 1.13 & 0.88 & 2.82 & 23.21 \\
\hline 5 & $22 / M$ & Craniopharyngioma & 9.26 & $5 \mathrm{~Gy} \times 4$ & 1.14 & 0.88 & 2.83 & 16.50 \\
\hline 6 & $24 / \mathrm{M}$ & Pituitary Adenoma & 3.53 & 5 Gy × 4 & 1.22 & 0.82 & 2.83 & 26.00 \\
\hline 7 & 45 / M & Pituitary Adenoma & 14.52 & $3.5 \mathrm{~Gy} \times 4$ & 1.09 & 0.91 & 2.87 & 30.36 \\
\hline 8 & $21 / F$ & Meningioma & 3.57 & $5 \mathrm{~Gy} \times 4$ & 1.14 & 0.87 & 2.96 & 17.00 \\
\hline 9 & $56 / \mathrm{M}$ & Meningioma & 59.12 & $5 \mathrm{~Gy} \times 4$ & 1.10 & 0.90 & 2.46 & 22.75 \\
\hline 10 & $60 / \mathrm{F}$ & $V^{\text {th }}$ nerve Schwannoma & 6.95 & $5 \mathrm{~Gy} \times 4$ & 1.15 & 0.86 & 3.19 & 22.70 \\
\hline
\end{tabular}
$340 \mathrm{~mm}^{3}$ to $59.12 \mathrm{~cm}^{3}$ ) was treated with clinically indicative doses (Table 4).

Table 4: Treatment characteristics of patient treated with extend system of Gamma Knife.

TV= Tumor volume, $\mathrm{CI}=$ Conformity index, $\mathrm{S}=$ Selectivity, GI= Gradient index, mTD= Minimum tumor dose 


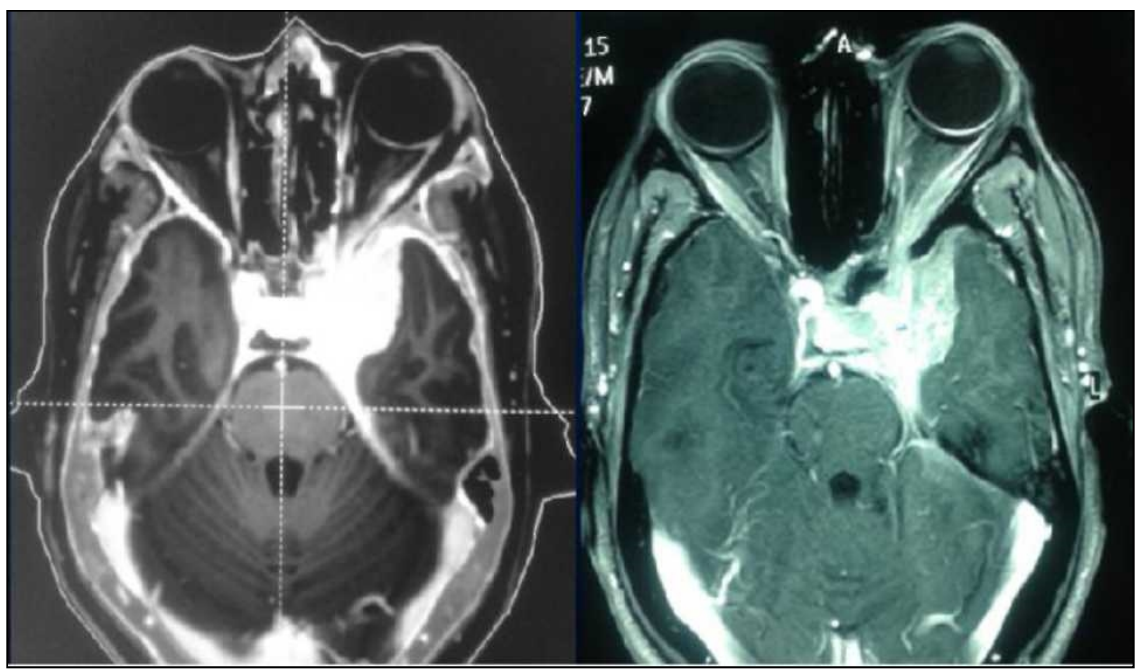

Figure 5 (a): A case of cavernous sinus meningioma; before and after treatment; Static tumor volume after 12 months follow-up.
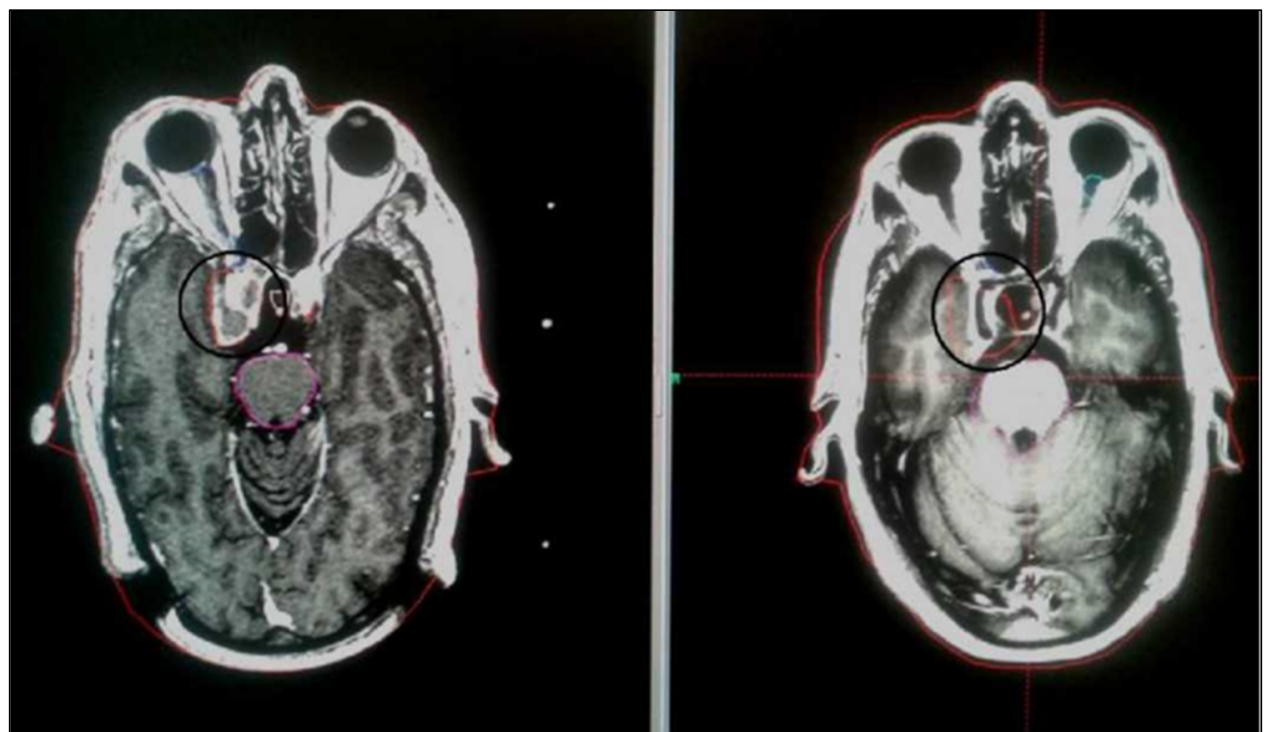

Figure 5 (b): A case of prolactinoma, before and after treatment; tumor size regression after 26 months follow-up.

\subsection{Dosimetric evaluation}

Multiple session of 3.5 Gy to 5.0 Gy per fraction results in cumulative dose of 14 Gy to 25 Gy at tumor margins. The coverage achieved was $91.91 \%$ (range, $90 \%$ to $95 \%$ ) of the tumor volume at $50 \%$ prescription isodose. The tumor has received mean dose of $66.76 \%$ (range, $63.0 \%$ to $71.8 \%$ ) compared to the dose maximum. The mean dose of $48.72 \%$ (range, $33.0 \%$ to $88.8 \%$ ) was recorded as a minimum dose received within the tumor volume (Table 4). Among various quality indices available in the literature, the mean of conformity index, selectivity and gradient index were observed as 1.30 (range, 1.09 to 2.40), 0.85 (range, 0.76 to 0.91 ) and 2.67 (range, 2.46 to 3.19 ) respectively for the tumors treated in this study.

Seven patients were available for follow-up at a median interval of 16 months (range, 9 months to 26 months).
All patients improved clinically with $100 \%$ radiographic control as there was no tumor progression on follow up MRI (Figure 5 a). One patient with prolactinoma, who underwent surgical decompression followed by fractionated radiosurgery (25 Gy in 5 fractions) exhibited significant reduction in tumor size after follow up of 26 months (Figure $5 \mathrm{~b}$ ). Three other patients with non-functional pituitary adenoma exhibited up to $50 \%$ reduction in the tumor size at a median follow up of 19 months (range, 16 months to 23 months) with no fresh clinical complaint.

\section{Discussion}

The extend system on Gamma Knife Perfexion is intended to deliver the radiation dose in a repeated series of lower dose fractions to the target volume. The ES could be used to treat target lesions neighbouring 
critical structures such as brain stem, chiasm, visual nerves and other radiosensitive nerves which were considered untreatable with Gamma Knife. ${ }^{11,17}$ First extend system physical performance study was done by Ruschin et al., ${ }^{18}$ to evaluate the immobilization ability of repositioning head frame (RHF). It was concluded that RHF is still susceptible to systematic uncertainties in anterior-posterior and superior-inferior directions. First clinical study was performed by Sayer et al. ${ }^{11}$ for treating four brain lesions. The study included treatment preparation for fractionated stereotactic radiosurgery using ES. Following this work Schlesinger et al. ${ }^{19}$, performed this treatment on ten patients and finally concluded that caution is to be taken while acquiring measurements specifically during patient's head rotation. In another study modified Winston-Lutz test was done by Lijun Ma et al. ${ }^{20}$ to determine the device accuracy of extend frame system. It was finally inferred in their study that a sub-millimeter radiological accuracy is clinically achievable for multi-session treatment.

To standardize the procedure and deposit reliable protocol, constant efforts have been made at each step of the process. Dentistry support in the preparation of upper dental impression with saliva suction stop ensures the aptness in creating mouth bite. A set of reference values for data sheet preparation, primarily on patient positioning system of Gamma Knife Perfexion assures the possibility of patient positioning without technical obstruction and comfort. The patient positional shift or RDV within the geometry of RCT is calculated manually using equation 1 and compared with auto generated values on system display (Table 3). An average variation of $0.004 \mathrm{~mm}$ (range, $0 \mathrm{~mm}$ to 0.005 $\mathrm{mm}$ ) was observed across all treatments. Insignificant variations on comparing manual to auto generated RDV values endorse auto generated calculations for the recording of patient positional shift. An entity ' $0.3 \mathrm{~mm}$ ' in resultant RDV with an average variation of $0.45 \mathrm{~mm}$ in a case of meningioma (volume $3.57 \mathrm{~cm}^{3}$, in Table 4), explicate the achievable positional accuracy in fractionated treatments. The factual positional shift causes the surrounding normal structure of the brain tissue exposed to redundant higher radiation dose. A vacuum level drop of $>10 \%$ on PSU indicates the substantial patient movement during imaging or treatment. The Gamma Knife machine pauses the treatment with an alarm automatically, whereas the patient imaging on CT machine has to discontinue manually at vacuum level drop. The treatment "pause" during circumstances draws repositioning of the patient to the reference position using RCT and rejoin treatment continuity. These instances of treatment pause were not observed during this study.

A comparative study of five patients was performed to check the variation in RDV calculations with two different assumptions (Table 3). The RDV with (1) measurement of three apertures per RCT panel and (2) maximum possible aperture measurement throughout RCT geometry was calculated. The second assumption improved an average RDV value by $0.062 \mathrm{~mm}$ (range, $0.031 \mathrm{~mm}$ to $0.110 \mathrm{~mm}$ ). A case with a maximum variation of $0.11 \mathrm{~mm}$ in comparative study supports improvising manufacturer's recommendations of three RCT aperture measurements for RDV calculations in fractionated stereotactic radiosurgery.

The patients treated with cumulative marginal dose of 14 Gy to 25 Gy in $4-5$ fractions of fractionated regime are generally well tolerated. The dose distributions in the radiation field favour the quality of an approved treatment plan through calculating conformity index, selectivity and gradient index. The mean of gradient index and selectivity of small tumor volumes $\left(<9.0 \mathrm{~cm}^{3}\right)$ are observed as 2.97 (range, 2.83 to 3.19 ) and 0.82 (range, 0.76 to 0.87 ), respectively, whereas it was 2.73 (range, 2.46 to 2.87 ) and 0.87 (range, 0.82 to 0.91 ) for larger tumor volumes. The gradient index for small and complex target volumes are comparatively higher to the larger tumor volume, however, consistency in selectivity shows similar proportion of the prescription isodose volume inside the tumor volume for all the cases. The maximum doses to most proximal critical structures like visual pathway and brain stem were observed as $10.3 \mathrm{~Gy}$ (range, 4.1 Gy to $12.1 \mathrm{~Gy}$ ) and $11.2 \mathrm{~Gy}$ (range, 3.5 Gy to $14.3 \mathrm{~Gy}$ ) respectively.

In brief, for the most promising results of fractionation with extend system of Gamma Knife, the study approached to four important steps: (i) justification on patient selection; clinical decision coupled with treatment feasibility; (ii) positional accuracy; an assurance of patient stability during the procedure; (3) exclusive dosimetric evaluation; an assurance of precise dose delivery; and (iv) an expected clinical outcome.

\section{Conclusion}

An approach extending the definition of fractionated radiosurgery is to make use of highly conformal treatments for larger and/ or irregular shaped tumor volumes neighbouring critical structures. The extend system provides excellent immobilization for multisession Gamma Knife stereotactic radiosurgery. An improved planning indices, reduced neighoubring structure doses and finally commendable clinical outcome demonstrate an efficacy of extend system for fractionated stereotactic radiosurgery.

The patient selection for the fractionated treatment with ES has limitations in treating patients with movable or irregular dental configuration. More clinical results would motivate to further improvise the method and dose delivery regimen for fractionated stereotactic radiosurgery with Gamma Knife. 


\section{Conflict of interest}

The authors declare that they have no conflicts of interest. The authors alone are responsible for the content and writing of the paper.

\section{Acknowledgement}

This study is supported by the research section of "All India Institute of Medical Sciences" New Delhi, India, as an intramural research project. [Project No A-247 at Gamma Knife Unit, Neurosciences centre, Department of Neurosurgery].

\section{References}

1. Firlik AD, Levy EI, Kondziolka D, et al. Staged volume radiosurgery followed by microsurgical resection: a novel treatment for giant cerebral arteriovenous malformations: technical case report. Neurosurgery. 1998;43:1223-8.

2. Haselsberger K, Maier T, Dominikus K et al. Staged gamma knife radiosurgery for large critically located benign meningiomas: evaluation of a series comprising 20 patients. J Neurol Neurosurg Psychiatry. 2009;80:1172-5.

3. Iwai Y, Yamanaka K, Nakajima H. Two-staged gamma knife radiosurgery for the treatment of large petroclival and cavernous sinus meningiomas. Surg Neurol. 2001;56:308-14.

4. Javalkar V, Pillai P, Vannemreddy P, et al. Gamma knife radiosurgery for arteriovenous malformations located in eloquent regions of the brain. Neurol India. 2009;57:617-21.

5. Kim JW, Im YS, Nam DH, et al. Preliminary report of multisession gamma knife radiosurgery for benign perioptic lesions: visual outcome in 22 patients. J Korean Neurosurg Soc. 2008;44:67- 71.

6. Niranjan A, Gobbel GT, Kondziolka D, et al. Experimental radiobiological investigations into radiosurgery: present understanding and future directions. Neurosurgery. 2004;55:495504.

7. Adler JR, Gibbs IC, Puataweepong P, et al. Visual field preservation after multisession cyberknife radiosurgery for perioptic lesions. Neurosurgery. 2008;62(Suppl 2):733-43.

8. Tuniz F, Soltys SG, Choi CY et al. Multisession Cyberknife stereotactic radiosurgery of large, benign cranial base tumors: preliminary study. Neurosurgery. 2009; 65: 898-907.

9. Milker-Zabel S, Huber P, Schlegel W, et al. Fractionated stereotactic radiation therapy in the management of primary optic nerve sheath meningiomas. J Neurooncol. 2009;94:419-24.

10. Simonova G, Novotny J, Novotny J Jr, et al. Fractionated stereotactic radiotherapy with the Leksell Gamma Knife: feasibility study. Radiother Oncol. 1995;37:108-16.

11. Sayer FT, Sherman JH, Yen CP, et al. Initial experience with the extend system: A relocatable frame system for multiple-session gamma knife radiosurgery. World Neurosurg. 2011;75(5-6):665-72.

12. Wells WM $3^{\text {rd }}$, Viola P, Atsumi H, et al. Multi-modal volume registration by maximization of mutual information. Med Image Anal. 1996;1(1):35-51.

13. Elia AE, Shih HA, Loeffler JS. Stereotactic radiation treatment for benign meningiomas. Neurosurg Focus. 2007;23(4):E5.

14. Killory BD, Kresl JJ, Wait SD, et al. Hypofractionated cyberknife radiosurgery for perichiasmatic pituitary adenomas: early results. Neurosurgery. 2009;64:A19-25.

15. Milker-Zabel S, Zabel-du Bois A, Huber P, et al. Fractionated stereotactic radiation therapy in the management of benign cavernous sinus meningiomas: long-term experience and review of the literature. Strahlenther Onkol. 2006; 182:635-40.

16. Park C, Papiez L, Zhang S, et al. Universal survival curve and single fraction equivalent dose: useful tools in understanding potency of ablative radiotherapy. Int J Radiat Oncol Biol Phys. 2008;70:847-52.

17. Devriendt D, De Smedt F, Glineur R, Massager N. Five-fraction gamma knife radiosurgery using the extend relocatable system for benign neoplasms close to optic pathways. Pract Radiat Oncol. 2015;5(3):119-25.

18. Ruschin M, Nayebi N, Carlsson P, et al. Performance of a novel repositioning head frame for Gamma Knife Perfexion and image-guided linac-based intracranial stereotactic radiotherapy. Int J Radiat Oncol Biol Phys. 2010;78:306-13.

19. Schlesinger D, Xu Z, Taylor F, et al. Interfraction and intrafraction performance of the gamma knife extend system for patient positioning and immobilization. J Neurosurg. 2012;117 suppl: 217-24.

20. Ma L, Pinnaduwage D, McDermotte M, Sneed PK. Whole-procedural radiological accuracy for delivering multi-session gamma knife radiosurgery with a relocatable frame system. Technol Cancer Res Treat. 2014;13(5)403-8. 